\title{
MULTIPLICITIES OF JUMPING POINTS FOR MIXED MULTIPLIER IDEALS
}

\author{
MARIA ALBERICH-CARRAMIÑANA, JOSEP ÀLVAREZ MONTANER, \\ FERRAN DACHS-CADEFAU, AND VÍCTOR GONZÁLEZ-ALONSO
}

\begin{abstract}
In this paper we make a systematic study of the multiplicity of the jumping points associated to the mixed multiplier ideals of a family of ideals in a complex surface with rational singularities. In particular we study the behaviour of the multiplicity by small perturbations of the jumping points. We also introduce a Poincaré series for mixed multiplier ideals and prove its rationality. Finally, we study the set of divisors that contribute to the log-canonical wall.
\end{abstract}

\section{INTRODUCTION}

Let $X$ be a complex surface with a rational singularity at a point $O \in X$ and $\mathcal{O}_{X, O}$ its corresponding local ring. Let $\mathfrak{a} \subseteq \mathcal{O}_{X, O}$ be an $\mathfrak{m}$-primary ideal where $\mathfrak{m}=\mathfrak{m}_{X, O}$ is the maximal ideal of $\mathcal{O}_{X, O}$. Then, for any real exponent $c>0$, we may consider its corresponding multiplier ideal $\mathcal{J}\left(\mathfrak{a}^{c}\right)$. Indeed, the multiplier ideals form a discrete nested sequence

$$
\mathcal{O}_{X, O} \supseteq \mathcal{J}\left(\mathfrak{a}^{\lambda_{1}}\right) \nsupseteq \mathcal{J}\left(\mathfrak{a}^{\lambda_{2}}\right) \nsupseteq \ldots \nsupseteq \mathcal{J}\left(\mathfrak{a}^{\lambda_{i}}\right) \nsupseteq \ldots
$$

indexed by an increasing sequence of rational numbers $0<\lambda_{1}<\lambda_{2}<\ldots$ such that $\mathcal{J}\left(\mathfrak{a}^{\lambda_{i}}\right)=\mathcal{J}\left(\mathfrak{a}^{c}\right) \nsupseteq \mathcal{J}\left(\mathfrak{a}^{\lambda_{i+1}}\right)$ for any $c \in\left[\lambda_{i}, \lambda_{i+1}\right)$. The $\lambda_{i}$ are the so-called jumping numbers of the ideal $\mathfrak{a}$. Ein, Lazarsfeld, Smith and Varolin [12], using the fact that the multiplier ideals are $\mathfrak{m}$-primary as well, defined the multiplicity of a point $c \in \mathbb{R}$ as

$$
m(c):=\operatorname{dim}_{\mathbb{C}} \frac{\mathcal{J}\left(\mathfrak{a}^{c-\varepsilon}\right)}{\mathcal{J}\left(\mathfrak{a}^{c}\right)}
$$

for $\varepsilon>0$ small enough. With this definition, it is clear that $c$ is a jumping number if and only if $m(c)>0$. A way to encode the information provided by the filtration of ideals (1.1) is by means of its Poincaré series of multiplier ideals

$$
P_{\mathfrak{a}}(t)=\sum_{c \in \mathbb{R}_{>0}} m(c) t^{c}
$$

All four authors are partially supported by Spanish Ministerio de Economía y Competitividad MTM201569135-P. VGA is partially supported by ERC StG 279723 Arithmetic of algebraic surfaces (SURFARI). MAC and JAM are also supported by Generalitat de Catalunya SGR2017-932 project and they are with the Barcelona Graduate School of Mathematics (BGSMath). MAC is also with the Institut de Robòtica i Informàtica Industrial (CSIC-UPC). 
introduced by Galindo and Montserrat in [14]. Actually, they proved that this series is a rational function when $X$ is smooth and $\mathfrak{a}$ is simple. In [3] we gave a systematic study of multiplicities and proved the rationality of the Poincaré series for any ideal $\mathfrak{a}$ in a complex surface with a rational singularity at $O$.

Whenever we extend to the case of mixed multiplier ideals $\mathcal{J}\left(\mathfrak{a}^{\mathbf{c}}\right):=\mathcal{J}\left(\mathfrak{a}_{1}^{c_{1}} \cdots \mathfrak{a}_{r}^{c_{r}}\right)$ associated to a tuple of $\mathfrak{m}$-primary ideals $\mathfrak{a}:=\left(\mathfrak{a}_{1}, \ldots, \mathfrak{a}_{r}\right) \subseteq\left(\mathcal{O}_{X, O}\right)^{r}$ and a point $\mathbf{c}:=$ $\left(c_{1}, \ldots, c_{r}\right)$ in the positive orthant $\mathbb{R}_{\geqslant 0}^{r}$, things become a little bit more trickier. Instead of having a partition of the positive real line into intervals defined by the jumping numbers where the multiplier ideals are constant, we get a partition of the positive real orthant into constancy regions whose boundary is described by the so-called jumping walls. We point out that only a few results on mixed multiplier ideals are available in the literature. Libgober and Mustaţă [19] studied properties of the log-canonical wall, i.e. the jumping wall associated to $\lambda_{0}=(0, \ldots, 0)$. Naie in [21] describes a nice property that jumping walls must satisfy. Cassou-Noguès and Libgober study in [10, 11] mixed multiplier ideals and jumping walls associated to germs of plane curves, under the analogous notion of ideals of quasi-adjunction and faces of quasi-adjunction (see [18]).

We may define the multiplicity of any given point $\boldsymbol{c} \in \mathbb{R}_{\geqslant 0}^{r}$ and we say that it is a jumping point, if and only if $m(\boldsymbol{c})>0$. In particular, jumping points lie on jumping walls. The most natural generalization of a Poincaré series for mixed multiplier ideals is to consider a filtration of ideals $\mathcal{J}\left(\mathfrak{a}^{\mathbf{c}}\right)$ indexed by points over a ray $L$ in $\mathbb{R}_{\geqslant 0}^{r}$ with direction vector in $\mathbb{Q}_{\geqslant 0}^{r}$ and set

$$
P_{\mathfrak{a}}(\underline{t} ; L)=\sum_{\boldsymbol{c} \in L} m(\boldsymbol{c}) \underline{t}^{\boldsymbol{c}}
$$

where $\underline{t}^{c}:=t_{1}^{c_{1}} \cdots t_{r}^{c_{r}}$. We have to mention that the multiplicity is sensitive to small perturbation of a given point. Indeed, if we consider a sequence of mixed multiplier ideals

$$
\mathcal{J}\left(\mathfrak{a}^{c_{0}}\right) \supsetneq \cdots \supsetneq \mathcal{J}\left(\mathfrak{a}^{\boldsymbol{c}_{i-1}}\right) \supsetneq \mathcal{J}\left(\mathfrak{a}^{c_{i}}\right) \supsetneq \mathcal{J}\left(\mathfrak{a}^{c_{i+1}}\right) \supsetneq \cdots
$$

indexed by jumping points $\left\{\boldsymbol{c}_{i}\right\}_{i \geqslant 0}$ over a ray $L$ and perturb minimally this ray, for example taking a parallel ray $L^{\prime}$ that is close enough, then the sequence of mixed multiplier ideals indexed by jumping points in $L^{\prime}$ may vary (see Example 4.1) and thus, the corresponding Poicaré series also varies.

The organization of the paper is as follows. In Section 2 we recall all the basics on mixed multiplier ideals. In Section 3 we extend the results of [3] to this setting. Namely, we make a systematic study of the multiplicities of points in the positive orthant. The main result is Theorem 3.4 where we give a precise formula for the multiplicity. We also prove in Theorem 3.9 that the Poincaré series associated to a ray is a rational function. In Section 4 we study the variation of the multiplicity of a jumping point by small perturbations. We prove that this multiplicity does not vary for points in the interior (with the Euclidean topology) of a $\mathcal{C}$-facet (see Proposition 4.2 ) but it does so in a controlled way at the intersection of $\mathcal{C}$-facets (see Theorem 4.5). In Section 5 we study the exceptional divisors that contribute to the log-canonical wall. Our main result is Theorem 5.9 where we 
establish (except for a very particular case considered in Proposition 5.7) a one-to-one correspondence between the facets of the log-canonical wall and the exceptional divisors of the so-called Newton nest (see Definition 5.1) generalizing a result of Cassou-Noguès and Libgober [11, Theorem 4.22].

\section{Mixed MUlTiplier ideAls}

Let $X$ be a complex surface with at most a rational singularity at a point $O \in X$ (see Artin [8] and Lipman [20] for details) and $\mathfrak{m}=\mathfrak{m}_{X, O}$ be the maximal ideal of the local ring $\mathcal{O}_{X, O}$ at $O$. Given a tuple of $\mathfrak{m}$-primary ideals $\mathfrak{a}=\left(\mathfrak{a}_{1}, \ldots, \mathfrak{a}_{r}\right) \subseteq\left(\mathcal{O}_{X, O}\right)^{r}$ we will consider a common log-resolution, that is, a birational morphism $\pi: X^{\prime} \rightarrow X$ such that $X^{\prime}$ is smooth, $\mathfrak{a}_{i} \cdot \mathcal{O}_{X^{\prime}}=\mathcal{O}_{X^{\prime}}\left(-F_{i}\right)$ for some effective Cartier divisors $F_{i}, i=1, \ldots, r$ and $\sum_{i=1}^{r} F_{i}+E$ is a divisor with simple normal crossings, where $E=\operatorname{Exc}(\pi)$ is the exceptional locus. Actually, the divisors $F_{i}$ are supported on the exceptional locus since the ideals are $\mathfrak{m}$-primary. The fundamental cycle is the unique smallest non-zero effective divisor $Z$ (with exceptional support) such that $Z \cdot E_{i} \leqslant 0$ for every $i=1, \ldots, r$. The fundamental cycle satisfies $\mathfrak{m} \cdot \mathcal{O}_{X^{\prime}}=\mathcal{O}_{X^{\prime}}(-Z)$ (see [8, Theorem 4]). We point out that any effective divisor with integer coefficients $\widetilde{D}$ is called antinef if $\widetilde{D} \cdot E_{i} \leqslant 0$ for every $i=1, \ldots, r$. Indeed, for any effective divisor $D$ there exists a unique minimal antinef divisor $\widetilde{D}$ satisfying $D \leqslant \widetilde{D}$ that is called the antinef closure of $D$. It can be computed using an inductive procedure called unloading (see [22] and [4] for details).

Since the point $O$ has a rational singularity, the exceptional locus $E$ is a tree of smooth rational curves $E_{1}, \ldots, E_{s}$. Moreover, the matrix of intersections $\left(E_{i} \cdot E_{j}\right)_{1 \leqslant i, j \leqslant s}$ is negative-definite. For any exceptional component $E_{j}$, we define the excess of $\mathfrak{a}_{i}$ at $E_{j}$ as $\rho_{i, j}=-F_{i} \cdot E_{j}$. We also recall the following notions:

- A component $E_{j}$ of $E$ is a rupture component if it intersects at least three more components of $E$ (different from $E_{j}$ ).

- We say that $E_{j}$ is dicritical if $\rho_{i, j}>0$ for some $i$. Such components correspond to Rees valuations (see [20]).

We define the mixed multiplier ideal at a point $\mathbf{c}:=\left(c_{1}, . ., c_{r}\right) \in \mathbb{R}_{\geqslant 0}^{r}$ as $^{1}$

$$
\mathcal{J}\left(\mathfrak{a}^{\mathbf{c}}\right):=\mathcal{J}\left(\mathfrak{a}_{1}^{c_{1}} \cdots \mathfrak{a}_{r}^{c_{r}}\right)=\pi_{*} \mathcal{O}_{X^{\prime}}\left(\left\lceil K_{\pi}-c_{1} F_{1}-\cdots-c_{r} F_{r}\right\rceil\right)
$$

where $\lceil\cdot\rceil$ denotes the round-up and the relative canonical divisor

$$
K_{\pi}=\sum_{i=1}^{s} k_{j} E_{j}
$$

is the $\mathbb{Q}$-divisor on $X^{\prime}$ supported on the exceptional locus $E$ characterized by the property $\left(K_{\pi}+E_{i}\right) \cdot E_{i}=-2$ for every exceptional component $E_{j}, j=1, \ldots, s$. We say that $X$ is log-canonical (resp. log-terminal) at $O$ if $k_{j} \geqslant-1$ ( resp. $\left.k_{j}>-1\right) \forall j$.

\footnotetext{
${ }^{1}$ By an abuse of notation, we will also denote $\mathcal{J}\left(\mathfrak{a}^{c}\right)$ its stalk at $O$ so we will omit the word "sheaf" if no
} confusion arises. 
Associated to any point $\boldsymbol{c} \in \mathbb{R}_{\geqslant 0}^{r}$, we consider:

. The region of $\boldsymbol{c}: \quad \mathcal{R}_{\mathfrak{a}}(\boldsymbol{c})=\left\{\boldsymbol{c}^{\prime} \in \mathbb{R}_{\geqslant 0}^{r} \mid \mathcal{J}\left(\mathfrak{a}^{\boldsymbol{c}^{\prime}}\right) \supseteq \mathcal{J}\left(\mathfrak{a}^{\boldsymbol{c}}\right)\right\}$

. The constancy region of $\boldsymbol{c}: \quad \mathcal{C}_{\mathfrak{a}}(\boldsymbol{c})=\left\{\boldsymbol{c}^{\prime} \in \mathbb{R}_{\geqslant 0}^{r} \mid \mathcal{J}\left(\mathfrak{a}^{c^{\prime}}\right)=\mathcal{J}\left(\mathfrak{a}^{c}\right)\right\}$

The boundary of the region $\mathcal{R}_{\mathfrak{a}}(\boldsymbol{c})$ is what we call the jumping wall associated to $\boldsymbol{c}$. One usually refers to the jumping wall of the origin as the log-canonical wall. It follows from the definition of mixed multiplier ideals that the jumping walls must lie on supporting hyperplanes of the form

$$
V_{j, \ell}: e_{1, j} z_{1}+\cdots+e_{r, j} z_{r}=\ell+k_{j}, \quad j=1, \ldots, s
$$

for a suitable $\ell \in \mathbb{Z}_{>0}$. Here we assume that the effective divisors $F_{i}$ such that $\mathfrak{a}_{i} \cdot \mathcal{O}_{X^{\prime}}=$ $\mathcal{O}_{X^{\prime}}\left(-F_{i}\right)$, for $i=1, \ldots, r$, are of the form $F_{i}=\sum_{j=1}^{s} e_{i, j} E_{j}$. Notice that each supporting hyperplane $V_{j, \ell}$ is associated to an exceptional component $E_{j}$. Indeed, we may find other exceptional components associated to the same hyperplane, that is, we may find $E_{i}$ and $\ell^{\prime} \in \mathbb{Z}_{>0}$ such that $V_{j, \ell}=V_{i, \ell^{\prime}}$.

It is proved in [4, Theorem 3.3] that the region $\mathcal{R}_{\mathfrak{a}}(\boldsymbol{c})$ is (the interior of) a rational convex polytope defined by the inequalities

$$
e_{1, j} z_{1}+\cdots+e_{r, j} z_{r}<k_{j}+1+e_{j}^{c}, \quad j=1, \ldots, s
$$

corresponding to either rupture or dicritical divisors $E_{j}$ and $D_{\boldsymbol{c}}=\sum e_{j}^{\boldsymbol{c}} E_{j}$ is the antinef closure of $\left\lfloor c_{1} F_{1}+\cdots+c_{r} F_{r}-K_{\pi}\right\rfloor$.

The intersection of the boundary of a connected component of a constancy region $\mathcal{C}_{\mathfrak{a}}(\boldsymbol{c})$ with a supporting hyperplane of $\mathcal{R}_{\mathfrak{a}}(\boldsymbol{c})$ is what we call a $\mathcal{C}$-facet of $\mathcal{C}_{\mathfrak{a}}(\boldsymbol{c})$. Every facet of a jumping wall decomposes into several $\mathcal{C}$-facets associated to different mixed multiplier ideals.

The main result of [4] is an algorithm to compute all the constancy regions, and their corresponding mixed multiplier ideals, in any desired range of the positive orthant $\mathbb{R}_{\geqslant 0}^{r}$. In particular the set of jumping walls of $\mathfrak{a}$, that we will denote from now on as $\mathbf{J W}_{\mathfrak{a}}$, is precisely described. The points on the jumping walls, which we will denote with $\lambda$ when we want to emphasize this fact, satisfy the property $\mathcal{J}\left(\mathfrak{a}^{\boldsymbol{c}}\right) \nsupseteq \mathcal{J}\left(\mathfrak{a}^{\boldsymbol{\lambda}}\right)$ for all $\boldsymbol{c} \in$ $\left\{\boldsymbol{\lambda}-\mathbb{R}_{\geqslant 0}^{r}\right\} \cap B_{\varepsilon}(\boldsymbol{\lambda})$ and $\varepsilon>0$ small enough. In the sequel, we will refer to these points as the jumping points of the tuple of ideals $\boldsymbol{a}$.

\section{Multiplicities of JUMPing POints}

In this section we are going to provide a systematic study of the multiplicity of any point $\boldsymbol{c} \in \mathbb{R}_{\geqslant 0}^{r}$. The results that we present are a natural generalization of the ones we obtained in [3].

Definition 3.1. Let $\mathfrak{a}=\left(\mathfrak{a}_{1}, \ldots, \mathfrak{a}_{r}\right) \subseteq\left(\mathcal{O}_{X, O}\right)^{r}$ be a tuple of $\mathfrak{m}$-primary ideals. We define the multiplicity attached to a point $\boldsymbol{c} \in \mathbb{R}_{\geqslant 0}^{r}$ as the codimension of $\mathcal{J}\left(\mathfrak{a}^{\boldsymbol{c}}\right)$ in $\mathcal{J}\left(\mathfrak{a}^{(1-\varepsilon) \boldsymbol{c}}\right)$ for $\varepsilon>0$ small enough, i.e. 


$$
m(\boldsymbol{c}):=\operatorname{dim}_{\mathbb{C}} \frac{\mathcal{J}\left(\mathfrak{a}^{(1-\varepsilon) \boldsymbol{c}}\right)}{\mathcal{J}\left(\mathbf{a}^{\boldsymbol{c}}\right)} .
$$

Our first goal is to compute explicitly these multiplicities using the theory of jumping divisors in this mixed multiplier ideals setting as considered in [4]. Since we are dealing with any general point, it will be more convenient to consider the notion of maximal jumping divisors as opposed to the minimal jumping divisors, which are only meaningful for jumping points.

Definition 3.2. Let $\mathfrak{a}=\left(\mathfrak{a}_{1}, \ldots, \mathfrak{a}_{r}\right) \subseteq\left(\mathcal{O}_{X, O}\right)^{r}$ be a tuple of ideals. Given any point $\boldsymbol{c} \in \mathbb{R}_{\geqslant 0}^{r}$, we define its maximal jumping divisor as the reduced divisor $H_{\boldsymbol{c}} \leqslant \sum_{i=1}^{r} F_{i}$ supported on those components $E_{j}$ such that

$$
c_{1} e_{1, j}+\cdots+c_{r} e_{r, j}-k_{j} \in \mathbb{Z}_{>0} .
$$

Equivalently, for a sufficiently small $\varepsilon>0$,

$$
H_{c}=\left\lceil K_{\pi}-(1-\varepsilon) c_{1} F_{1}-\cdots-(1-\varepsilon) c_{r} F_{r}\right\rceil-\left\lceil K_{\pi}-c_{1} F_{1}-\cdots-c_{r} F_{r}\right\rceil .
$$

In particular, we have

$$
\mathcal{J}\left(\mathfrak{a}^{(1-\varepsilon) \boldsymbol{c}}\right)=\pi_{*} \mathcal{O}_{X^{\prime}}\left(\left\lceil K_{\pi}-c_{1} F_{1}-\cdots-c_{r} F_{r}\right\rceil+H_{\boldsymbol{c}}\right) .
$$

The following numerical properties for maximal jumping divisors will be useful for our purposes. We will skip the details of the proof just because it is a natural generalization of [3, Proposition 3.6] and the same proof holds mutatis mutandi.

Proposition 3.3. Fix any $\boldsymbol{c} \in \mathbb{R}_{\geqslant 0}^{r}$, and let $H_{\boldsymbol{c}}$ be its associated maximal jumping divisor. Then the following inequalities hold:

- $\left(\left\lceil K_{\pi}-c_{1} F_{1}-\cdots-c_{r} F_{r}\right\rceil+H_{c}\right) \cdot E_{i} \geqslant-1$ for all $E_{i} \leqslant H_{c}$, and

- $\left(\left\lceil K_{\pi}-c_{1} F_{1}-\cdots-c_{r} F_{r}\right\rceil+H_{\boldsymbol{c}}\right) \cdot H \geqslant-1$ for any connected component $H \leqslant H_{\boldsymbol{c}}$.

The main result of this section is the following:

Theorem 3.4. Let $\mathfrak{a}=\left(\mathfrak{a}_{1}, \ldots, \mathfrak{a}_{r}\right) \subseteq\left(\mathcal{O}_{X, O}\right)^{r}$ be a tuple of $\mathfrak{m}$-primary ideals and $H_{\boldsymbol{c}}$ the maximal jumping divisor associated to some $\boldsymbol{c} \in \mathbb{R}_{\geqslant 0}^{r}$. Then,

$$
m(\boldsymbol{c})=\left(\left\lceil K_{\pi}-c_{1} F_{1}-\cdots-c_{r} F_{r}\right\rceil+H_{\boldsymbol{c}}\right) \cdot H_{\boldsymbol{c}}+\#\left\{\text { connected components of } H_{\boldsymbol{c}}\right\} .
$$

Proof. Given the short exact sequence

$$
\begin{gathered}
0 \longrightarrow \mathcal{O}_{X^{\prime}}\left(\left\lceil K_{\pi}-c_{1} F_{1}-\cdots-c_{r} F_{r}\right\rceil\right) \longrightarrow \mathcal{O}_{X^{\prime}}\left(\left\lceil K_{\pi}-c_{1} F_{1}-\cdots-c_{r} F_{r}\right\rceil+H_{c}\right) \longrightarrow \\
\longrightarrow \mathcal{O}_{H_{\boldsymbol{c}}}\left(\left\lceil K_{\pi}-c_{1} F_{1}-\cdots-c_{r} F_{r}\right\rceil+H_{\boldsymbol{c}}\right) \longrightarrow 0
\end{gathered}
$$

we have, after pushing it forward to $X$ and applying local vanishing [17] for the case of mixed multiplier ideals

$$
\begin{aligned}
0 \longrightarrow \mathcal{J}\left(\mathfrak{a}^{\boldsymbol{c}}\right) \longrightarrow \mathcal{J}\left(\mathfrak{a}^{(1-\varepsilon) \boldsymbol{c}}\right) & \longrightarrow \\
& \longrightarrow H^{0}\left(H_{\boldsymbol{c}}, \mathcal{O}_{H_{\boldsymbol{c}}}\left(\left\lceil K_{\pi}-c_{1} F_{1}-\cdots-c_{r} F_{r}\right\rceil+H_{\boldsymbol{c}}\right)\right) \otimes \mathbb{C}_{O} \longrightarrow 0
\end{aligned}
$$


for $\varepsilon$ small enough. Therefore the multiplicity of $\boldsymbol{c}$ is just

$$
\begin{aligned}
m(\boldsymbol{c}) & =h^{0}\left(H_{\boldsymbol{c}}, \mathcal{O}_{H_{\boldsymbol{c}}}\left(\left\lceil K_{\pi}-c_{1} F_{1}-\cdots-c_{r} F_{r}\right\rceil+H_{\boldsymbol{c}}\right)\right) \\
& =\sum_{E_{i} \leqslant H_{\boldsymbol{c}}} h^{0}\left(E_{i}, \mathcal{O}_{E_{i}}\left(\left\lceil K_{\pi}-c_{1} F_{1}-\cdots-c_{r} F_{r}\right\rceil+H_{\boldsymbol{c}}\right)\right)-a_{H_{\boldsymbol{c}}},
\end{aligned}
$$

where in the second equality we have used that $H_{c}$ has simple normal crossings, and hence the sections of the line bundle $\mathcal{O}_{H_{\boldsymbol{c}}}\left(\left\lceil K_{\pi}-c_{1} F_{1}-\cdots-c_{r} F_{r}\right\rceil+H_{\boldsymbol{c}}\right)$ correspond to sections over each component that agree on the $a_{H_{c}}$ intersections, where $a_{H_{c}}$ denotes the number of edges of $H_{\boldsymbol{c}}$ in the dual graph. Then, since we have

$$
\operatorname{deg} \mathcal{O}_{E_{i}}\left(\left\lceil K_{\pi}-c_{1} F_{1}-\cdots-c_{r} F_{r}\right\rceil+H_{\boldsymbol{c}}\right)=\left(\left\lceil K_{\pi}-c_{1} F_{1}-\cdots-c_{r} F_{r}\right\rceil+H_{\boldsymbol{c}}\right) \cdot E_{i} \geqslant-1
$$

by Proposition 3.3, we get

$$
\begin{aligned}
m(\boldsymbol{c}) & =\sum_{E_{i} \leqslant H_{\boldsymbol{c}}}\left(\left(\left\lceil K_{\pi}-c_{1} F_{1}-\cdots-c_{r} F_{r}\right\rceil+H_{\boldsymbol{c}}\right) \cdot E_{i}+1\right)-a_{H_{\boldsymbol{c}}} \\
& =\left(\left\lceil K_{\pi}-c_{1} F_{1}-\cdots-c_{r} F_{r}\right\rceil+H_{\boldsymbol{c}}\right) \cdot H_{\boldsymbol{c}}+v_{H_{\boldsymbol{c}}}-a_{H_{\boldsymbol{c}}} \\
& =\left(\left\lceil K_{\pi}-c_{1} F_{1}-\cdots-c_{r} F_{r}\right\rceil+H_{\boldsymbol{c}}\right) \cdot H_{\boldsymbol{c}}+\#\left\{\text { connected components of } H_{\boldsymbol{c}}\right\} .
\end{aligned}
$$

The above formula can be rephrased as follows

Corollary 3.5. Let $\mathfrak{a}=\left(\mathfrak{a}_{1}, \ldots, \mathfrak{a}_{r}\right) \subseteq\left(\mathcal{O}_{X, O}\right)^{r}$ be a tuple of $\mathfrak{m}$-primary ideals and $H_{\boldsymbol{c}}$ the maximal jumping divisor associated to some $\boldsymbol{c} \in \mathbb{R}_{\geqslant 0}^{r}$. Then,

$$
\begin{aligned}
m(\boldsymbol{c})= & \sum_{E_{i} \leqslant H_{\boldsymbol{c}}}\left(\sum_{E_{j} \in \operatorname{Adj}\left(E_{i}\right)}\left\{c_{1} e_{1, j}+\cdots+c_{r} e_{r, j}-k_{j}\right\}+c_{1} \rho_{1, i}+\cdots+c_{r} \rho_{r, i}\right) \\
& -\#\left\{\text { connected components of } H_{\boldsymbol{c}}\right\} .
\end{aligned}
$$

We may also provide a very simple numerical criterion to detect whether a given point $c \in \mathbb{R}_{>0}^{r}$ is a jumping point.

Theorem 3.6. Let $\mathfrak{a}=\left(\mathfrak{a}_{1}, \ldots, \mathfrak{a}_{r}\right) \subseteq\left(\mathcal{O}_{X, O}\right)^{r}$ be a tuple of $\mathfrak{m}$-primary ideals and $\boldsymbol{c} \in \mathbb{R}_{\geqslant 0}^{r}$. Then, $\boldsymbol{c}$ is a jumping point if and only if $m(\boldsymbol{c})>0$ or equivalently, there exists a connected component $H \leqslant H_{c}$ such that

$$
\left(\left\lceil K_{\pi}-c_{1} F_{1}-\cdots-c_{r} F_{r}\right\rceil+H_{c}\right) \cdot H \geq 0 .
$$

Proof. We have

$$
\begin{aligned}
m(\boldsymbol{c}) & =\left(\left\lceil K_{\pi}-c_{1} F_{1}-\cdots-c_{r} F_{r}\right\rceil+H_{\boldsymbol{c}}\right) \cdot H_{\boldsymbol{c}}+\#\left\{\text { connected components of } H_{\boldsymbol{c}}\right\} \\
& =\sum_{H \leqslant H_{\boldsymbol{c}}}\left(\left(\left\lceil K_{\pi}-c_{1} F_{1}-\cdots-c_{r} F_{r}\right\rceil+H_{\boldsymbol{c}}\right) \cdot H+1\right),
\end{aligned}
$$

where the sum is taken over all the connected components $H \leqslant H_{\boldsymbol{c}}$. The result follows since we have $\left(\left\lceil K_{\pi}-c_{1} F_{1}-\cdots-c_{r} F_{r}\right\rceil+H_{c}\right) \cdot H \geqslant-1$ by Proposition 3.3. 
3.1. Poincaré series of mixed multiplier ideals. Given a m-primary ideal $\mathfrak{a} \subseteq \mathcal{O}_{X, O}$ we consider its Poincaré series

$$
P_{\mathfrak{a}}(t)=\sum_{c \in \mathbb{R}_{\geqslant 0}} m(c) t^{c}
$$

which was first considered, in the case that $X$ is smooth and $\mathfrak{a}$ is simple, by Galindo and Montserrat [14] and extended in [3] to the case where $X$ has a rational singularity and $\mathfrak{a}$ is any $\mathfrak{m}$-primary ideal.

For a tuple of $\mathfrak{m}$-primary ideals $\mathfrak{a}=\left\{\mathfrak{a}_{1}, \ldots, \mathfrak{a}_{r}\right\} \subseteq\left(\mathcal{O}_{X, O}\right)^{r}$ we are going to give a generalization of this series by considering a sequence of mixed multiplier ideals indexed by points in a ray $L: \boldsymbol{c}_{0}+\mu \mathbf{u}$ in the positive orthant $\mathbb{R}_{\geqslant 0}^{r}$ with $\mathbf{u}=\left(u_{1}, \ldots, u_{r}\right) \in \mathbb{Z}_{\geqslant 0}^{r}$, $\mathbf{u} \neq \mathbf{0}$ and $\boldsymbol{c}_{0} \in \mathbb{Q}_{\geqslant 0}^{r}$. Here we are considering, for simplicity, a point $\boldsymbol{c}_{0}$ belonging to a coordinate hyperplane but not necessarily being the origin and $\mu \in \mathbb{R}_{\geqslant 0}$. Namely, we consider the sequence of mixed multiplier ideals

$$
\mathcal{J}\left(\mathfrak{a}^{c_{0}}\right) \supsetneq \mathcal{J}\left(\mathfrak{a}^{c_{1}}\right) \supsetneq \mathcal{J}\left(\mathfrak{a}^{c_{2}}\right) \supsetneq \cdots \supsetneq \mathcal{J}\left(\mathfrak{a}^{\boldsymbol{c}_{i}}\right) \supsetneq \cdots
$$

where $\left\{\boldsymbol{c}_{i}\right\}_{i>0}=L \cap \mathbf{J} \mathbf{W}_{\mathfrak{a}}$ or equivalently $\left\{\boldsymbol{c}_{i}\right\}_{i>0}$ is the set of jumping points of this sequence. Then we define the Poincaré series of $\mathfrak{a}$ alongside the ray $L$ as

$$
P_{\mathfrak{a}}(\underline{t} ; L)=\sum_{\boldsymbol{c} \in L} m(\boldsymbol{c}) \underline{t}^{c} .
$$

where $\underline{t}^{c}:=t_{1}^{c_{1}} \cdots t_{r}^{c_{r}}$. Notice that we have

$$
P_{\mathfrak{a}}(\underline{t} ; L)=\sum_{\boldsymbol{c} \in L} m(\boldsymbol{c}) \underline{t}^{\boldsymbol{c}}=\sum_{\boldsymbol{c} \in\left[\boldsymbol{c}_{0}, \boldsymbol{c}_{0}+\mathbf{u}\right)} \sum_{k \in \mathbb{N}} m(\boldsymbol{c}+k \mathbf{u}) \underline{\boldsymbol{c}}^{\boldsymbol{c}+k \mathbf{u}}=\underline{t}^{\boldsymbol{c}_{0}} \sum_{\mu \in[0,1)} \sum_{k \in \mathbb{N}} m(\boldsymbol{c}+k \mathbf{u}) \underline{t}^{(\mu+k) \mathbf{u}}
$$

where the last equality follows from the fact that we are considering points of the form $\boldsymbol{c}=\boldsymbol{c}_{0}+\mu \mathbf{u}$ with $\mu \in[0,1)$. Our goal is to prove that this Poincaré series is rational in the sense that it belongs to the field of fractional functions $\mathbb{C}\left(z_{1}, \ldots, z_{r}\right)$, where the indeterminate $z_{i}$ corresponds to a fractional power $t_{i}^{1 / e}$ for $e \in \mathbb{N}_{>0}$ being the least common multiple of the denominators of the coordinates of all jumping points. To do so we need to prove a linear recurrence among the coefficients of the series. A key ingredient will be a periodicity property of the maximal jumping divisor which follows from its definition.

Lemma 3.7. For any $\boldsymbol{c} \in \mathbb{R}_{>0}^{r}$ and $\boldsymbol{\alpha}=\left(\alpha_{1}, \ldots, \alpha_{r}\right) \in \mathbb{Z}_{\geqslant 0}^{r}$ we have $H_{\boldsymbol{c}}=H_{\boldsymbol{c}+\boldsymbol{\alpha}}$.

The linear recurrence that the multiplicities satisfy is described in terms of the excesses at dicritical components.

Proposition 3.8. Let $\mathfrak{a}=\left(\mathfrak{a}_{1}, \ldots, \mathfrak{a}_{r}\right) \subseteq\left(\mathcal{O}_{X, O}\right)^{r}$ be a tuple of $\mathfrak{m}$-primary ideals and $\boldsymbol{\alpha}=\left(\alpha_{1}, \ldots, \alpha_{r}\right) \in \mathbb{Z}_{\geqslant 0}^{r}$. Then,

$$
m(\boldsymbol{c}+\boldsymbol{\alpha})-m(\boldsymbol{c})=\sum_{E_{i} \leqslant H_{\boldsymbol{c}}} \sum_{j=1}^{r} \alpha_{j} \rho_{j, i} .
$$


Proof. Lemma 3.7 above states that $\boldsymbol{c}$ and $\boldsymbol{c}+\boldsymbol{\alpha}$ have the same maximal jumping divisor, say $H_{\boldsymbol{c}}$. Therefore, by Theorem 3.4, we have

$$
m(\boldsymbol{c}+\boldsymbol{\alpha})-m(\boldsymbol{c})=-\left(\alpha_{1} F_{1}+\cdots+\alpha_{r} F_{r}\right) \cdot H_{\boldsymbol{c}}=\sum_{E_{i} \leqslant H_{\boldsymbol{c}}} \sum_{1 \leqslant j \leqslant r} \alpha_{j} \rho_{j, i} .
$$

In the sequel, we will just denote $\rho_{\boldsymbol{c}, \boldsymbol{\alpha}}:=\sum_{E_{i} \leqslant H_{\boldsymbol{c}}} \sum_{j=1}^{r} \alpha_{j} \rho_{j, i}$. The formula for the Poincaré series that we obtain is the following:

Theorem 3.9. Let $\mathfrak{a}=\left\{\mathfrak{a}_{1}, \ldots, \mathfrak{a}_{r}\right\} \subseteq\left(\mathcal{O}_{X, O}\right)^{r}$ be a tuple of $\mathfrak{m}$-primary ideals and let $L: \boldsymbol{c}_{0}+\mu \mathbf{u}$ be a ray in the positive orthant $\mathbb{R}_{\geqslant 0}^{r}$ with $\mathbf{u} \in \mathbb{Z}_{\geqslant 0}, \mathbf{u} \neq \mathbf{0}$. The Poincaré series of $\mathfrak{a}$ alongside $L$ can be expressed as

$$
P_{\mathfrak{a}}(\underline{t} ; L)=\underline{t}^{\boldsymbol{c}_{0}} \sum_{\mu \in[0,1)}\left(\frac{m\left(\boldsymbol{c}_{0}+\mu \mathbf{u}\right)}{1-\underline{t}^{\mathbf{u}}}+\rho_{\boldsymbol{c}_{0}+\mu \mathbf{u}, \mathbf{u}} \frac{\underline{t}^{\mathbf{u}}}{\left(1-\underline{t}^{\mathbf{u}}\right)^{2}}\right) \underline{t}^{\mu \mathbf{u}}
$$

Proof. Given a point $\boldsymbol{c}=\boldsymbol{c}_{0}+\mu \mathbf{u}$, with $\mu \in[0,1)$ we have, using Proposition 3.8, that

$$
m(\boldsymbol{c}+k \mathbf{u})=m(\boldsymbol{c})+k \sum_{E_{i} \leqslant H_{\boldsymbol{c}}} \sum_{j=1}^{r} u_{j} \rho_{j, i}=m(\boldsymbol{c})+k \rho_{\boldsymbol{c}, \mathbf{u}}
$$

Therefore

$$
\begin{aligned}
\sum_{k \geqslant 0} m(\boldsymbol{c}+k \mathbf{u}) \underline{t}^{\boldsymbol{c}+k \mathbf{u}} & =m(\boldsymbol{c}) \underline{t}^{\boldsymbol{c}}+\left(m(\boldsymbol{c})+\rho_{\boldsymbol{c}, \mathbf{u}}\right) \underline{t}^{\boldsymbol{c}+\mathbf{u}}+\left(m(\boldsymbol{c})+2 \rho_{\boldsymbol{c}, \mathbf{u}}\right) \underline{t}^{\boldsymbol{c}+2 \mathbf{u}}+\cdots \\
& =\left(\frac{m(\boldsymbol{c})}{1-\underline{t}^{\mathbf{u}}}+\rho_{\boldsymbol{c}, \mathbf{u}} \frac{\underline{t}}{\left(1-\underline{t}^{\mathbf{u}}\right)^{2}}\right) \underline{t}^{\boldsymbol{c}}
\end{aligned}
$$

and the result follows.

Remark 3.10. In the case that $L$ is the $i$-th axis of the positive orthant $\mathbb{R}_{\geqslant 0}^{r}$, in particular if $\boldsymbol{c}_{0}$ is the origin, we obtain the Poincaré series of the ideal $\mathfrak{a}_{i}$.

\section{Multiplicities of JUMPing POINTS AFter SMAll PERTURBATIONS}

Let $\mathfrak{a}=\left(\mathfrak{a}_{1}, \ldots, \mathfrak{a}_{r}\right) \subseteq\left(\mathcal{O}_{X, O}\right)^{r}$ be a tuple of $\mathfrak{m}$-primary ideals and consider two parallel rays $L: \boldsymbol{c}_{0}+\mu \mathbf{u}$ and $L^{\prime}: \boldsymbol{c}_{0}^{\prime}+\mu \mathbf{u}$ as those considered in the previous section that are close enough. Our aim is to compare the sequences of mixed multiplier ideals indexed by points in both rays and see how the multiplicity of a jumping point varies with a small perturbation. To illustrate this phenomenon we start with the following example.

Example 4.1. Consider the tuple of ideals $\mathfrak{a}=\left(\mathfrak{a}_{1}, \mathfrak{a}_{2}\right)$ on a smooth surface $X$ given by:

$$
\begin{aligned}
& \cdot \mathfrak{a}_{1}=\left((x+y)^{4}, x^{9}(x+y), x^{11}, x^{6}(x+y)^{2}, x^{3}(x+y)^{3}\right), \\
& \cdot \mathfrak{a}_{2}=\left(y^{3}, x^{7}, x^{5} y, x^{3} y^{2}\right) .
\end{aligned}
$$

The dual graph of the log-resolution of $\mathfrak{a}$ is as follows: 


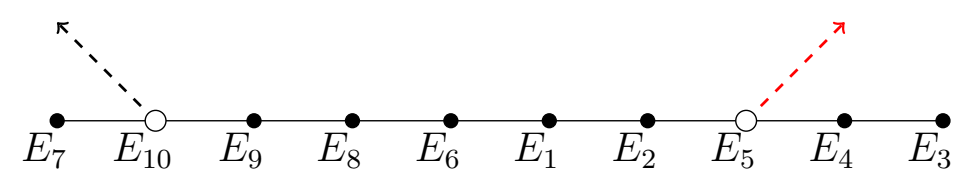

where the blank dots correspond to dicritical divisors and their excesses are represented by broken arrows. The divisors associated with this resolution are

$\cdot F_{1}=4 E_{1}+4 E_{2}+4 E_{3}+8 E_{4}+12 E_{5}+8 E_{6}+11 E_{7}+20 E_{8}+32 E_{9}+44 E_{10}$,

$\cdot F_{2}=3 E_{1}+6 E_{2}+7 E_{3}+14 E_{4}+21 E_{5}+3 E_{6}+3 E_{7}+6 E_{8}+9 E_{9}+12 E_{10}$

and the relative canonical divisor is:

$$
\cdot K_{\pi}=E_{1}+2 E_{2}+3 E_{3}+6 E_{4}+9 E_{5}+2 E_{6}+3 E_{7}+6 E_{8}+10 E_{9}+14 E_{10} .
$$

In Figure 2, we present the constancy regions of the corresponding mixed multiplier ideals, those regions are computed using the algorithm in [4]. The chains of mixed multiplier ideals over the parallel rays $L:\left(0, \frac{101}{780}\right)+\mu(1,1)$ and $L^{\prime}:\left(0, \frac{37}{390}\right)+\mu(1,1)$ are given in Table 1. The sets of generators for these ideals are computed using the algorithm in [1] (see also [9] ).

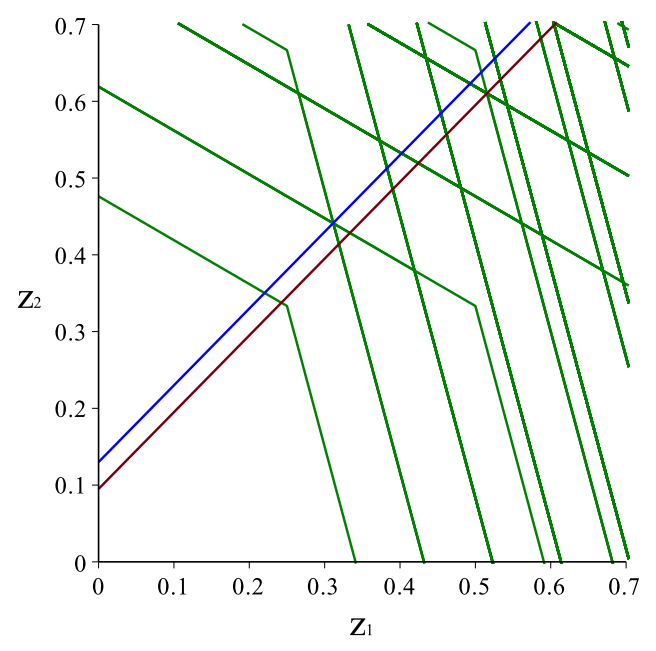

Figure 1. Constancy regions of the mixed multiplier ideals of $\mathfrak{a}$ and the rays $L, L^{\prime}$, in blue and Bordeaux respectively.

In the previous example, we observe that the chains of mixed multiplier ideals differ whenever the corresponding ray crosses the intersection of $\mathcal{C}$-facets. Indeed, the multiplicity of a jumping point at the intersection of $\mathcal{C}$-facets is bigger than the multiplicities of jumping points in its neighborhood. The aim of this section is to provide an explanation to this phenomenon. We start with the fact that the multiplicity does not increase in the interior of $\mathcal{C}$-facets.

Proposition 4.2. Let $\mathfrak{a}=\left(\mathfrak{a}_{1}, \ldots, \mathfrak{a}_{r}\right) \subseteq\left(\mathcal{O}_{X, O}\right)^{r}$ be a tuple of $\mathfrak{m}$-primary ideals and let $\boldsymbol{\lambda}, \boldsymbol{\lambda}^{\prime}$ be two jumping points in the interior of a $\mathcal{C}$-facet. Then $m(\boldsymbol{\lambda})=m\left(\boldsymbol{\lambda}^{\prime}\right)$. 


\begin{tabular}{|l|l|l|l|}
\hline $\begin{array}{l}\text { Jumping } \\
\text { points in } L\end{array}$ & $\mathcal{J}\left(\mathfrak{a}^{\boldsymbol{\lambda}}\right)$ & $\begin{array}{l}\text { Jumping } \\
\text { points in } L^{\prime}\end{array}$ & $\mathcal{J}\left(\mathfrak{a}^{\boldsymbol{\lambda}}\right)$ \\
\hline \hline$\left(\frac{81}{260}, \frac{86}{195}\right)$ & $(x, y)$ & $\left(\frac{67}{130}, \frac{119}{195}\right)$ & $(x, y)$ \\
\hline \multicolumn{2}{|l|}{$\left(\frac{203}{520}, \frac{757}{1560}\right)$} & $\left(x+y, x^{2}\right)$ \\
\hline$\left(\frac{\mathbf{6 3 1}}{\mathbf{2 8 6 0}}, \frac{\mathbf{7 5 1}}{\mathbf{2 1 4 5}}\right)$ & $\left(x^{2}, y^{2}, x y\right)$ & $\left(\frac{267}{455}, \frac{1861}{2730}\right)$ & $\left(x^{2}, y^{2}, x y\right)$ \\
\hline$\left(\frac{697}{1820}, \frac{1399}{2730}\right)$ & $\left(x y+x^{2}, x^{3}, y^{2}+2 x y+x^{2}\right)$ & $\left(\frac{347}{1430}, \frac{724}{2145}\right)$ & $\left(x y+x^{2}, x^{3}, y^{2}+2 x y+x^{2}\right)$ \\
\hline$\left(\frac{827}{1820}, \frac{797}{1365}\right)$ & $\left(y^{2}+x y, x^{2} y, x^{2} y+x^{3}\right)$ & $\left(\frac{477}{1430}, \frac{919}{2145}\right)$ & $\left(y^{2}+x y, x^{2} y, x^{2} y+x^{3}\right)$ \\
\hline$\left(\frac{957}{1820}, \frac{1789}{2730}\right)$ & $\left(y^{2}+x y, x^{2} y, x^{2} y+x^{3}\right)$ & $\left(\frac{607}{1430}, \frac{1114}{2145}\right)$ & $\left(y^{2}+x y, x^{2} y, x^{2} y+x^{3}\right)$ \\
\hline$\left(\frac{1151}{2860}, \frac{1141}{2145}\right)$ & $\left(y^{2}+x y, x^{3} y, x^{3} y+x^{4}\right)$ & \multicolumn{2}{|l|}{} \\
\hline$\left(\frac{1411}{2860}, \frac{1336}{2145}\right)$ & $\begin{array}{l}\left(y^{3}+x y^{2}, x^{3} y, x^{3} y+x^{4}, x^{2} y^{2},\right. \\
\left.x y^{2}+x^{2} y\right)\end{array}$ & $\left(\frac{\mathbf{1 1 6 1}}{\left.\mathbf{3 6 4 0}, \frac{\mathbf{4 5 1 9}}{\mathbf{1 0 9 2 0}}\right)}\right.$ & $\begin{array}{l}\left(y^{3}+x y^{2}, x^{3} y, x^{3} y+x^{4}, x^{2} y^{2},\right. \\
\left.x y^{2} y\right)\end{array}$ \\
\hline$\left(\frac{1849}{3640}, \frac{6961}{10920}\right)$ & $\begin{array}{l}\left(y^{3}+2 x y^{2}+x^{2} y, x^{4} y, x y^{2}+\right. \\
x^{2} y, \\
\left.x^{2} y^{2}+2 x^{3} y+x^{4}, x^{3} y+x^{4}\right)\end{array}$ & $\left(\frac{1681}{3640}, \frac{6079}{10920}\right)$ & $\begin{array}{l}\left(y^{3}+2 x y^{2}+x^{2} y, x^{4} y, x y^{2}+\right. \\
x^{2} y, \\
\left.x^{2} y^{2}+2 x^{3} y+x^{4}, x^{3} y+x^{4}\right)\end{array}$ \\
\hline$\left(\frac{2109}{3640}, \frac{7741}{10920}\right)$ & $\begin{array}{l}\left(y^{3}+2 x y^{2}+x^{2} y, x^{2} y^{2}+x^{3} y,\right. \\
\left.x^{4} y, x^{2} y^{2}+2 x^{3} y+x^{4}\right)\end{array}$ & $\left(\frac{1941}{3640}, \frac{6859}{10920}\right)$ & $\begin{array}{l}\left(y^{3}+2 x y^{2}+x^{2} y, x^{2} y^{2}+x^{3} y,\right. \\
\left.x^{4} y, x^{2} y^{2}+2 x^{3} y+x^{4}\right)\end{array}$ \\
\hline$\left(\frac{2369}{3640}, \frac{8521}{10920}\right)$ & $\begin{array}{l}\left(y^{3}+2 x y^{2}+x^{2} y, x^{2} y^{2}+x^{3} y,\right. \\
\left.x^{5} y, x^{2} y^{2}+2 x^{3} y+x^{4}\right)\end{array}$ & $\left(\frac{2201}{3640}, \frac{7639}{10920}\right)$ & $\begin{array}{l}\left(y^{3}+2 x y^{2}+x^{2} y, x^{2} y^{2}+x^{3} y,\right. \\
\left.x^{5} y, x^{2} y^{2}+2 x^{3} y+x^{4}\right)\end{array}$ \\
\hline
\end{tabular}

TABLE 1. Chains of mixed multiplier ideals of $\mathfrak{a}$ over the rays $L$ and $L^{\prime}$. In bold type we present the jumping points with multiplicity 2 .

To prove this result it is more convenient to compute the multiplicity of a jumping point by using the so-called minimal jumping divisor instead of the maximal jumping divisor as we did in Section 3. This minimal jumping divisor is closely related to the algorithm developed in [4] to compute the constancy regions of mixed multiplier ideals. We give its definition below but we refer to [4] for details.

Definition 4.3. Let $\mathfrak{a}=\left(\mathfrak{a}_{1}, \ldots, \mathfrak{a}_{r}\right) \subseteq\left(\mathcal{O}_{X, O}\right)^{r}$ be a tuple of ideals. Given a jumping point $\boldsymbol{\lambda}=\left(\lambda_{1}, \ldots, \lambda_{r}\right) \in \mathbb{R}_{\geqslant 0}^{r}$, its corresponding minimal jumping divisor is the reduced divisor $G_{\lambda} \leqslant \sum_{i=1}^{r} F_{i}$ supported on those components $E_{j}$ for which the point $\lambda$ satisfies

$$
\lambda_{1} e_{1, j}+\cdots+\lambda_{r} e_{r, j}=k_{j}+1+e_{j}^{(1-\varepsilon) \lambda},
$$

where, for a sufficiently small $\varepsilon>0, D_{(1-\varepsilon) \boldsymbol{\lambda}}=\sum e_{j}^{(1-\varepsilon) \boldsymbol{\lambda}} E_{j}$ is the antinef closure of

$$
\left\lfloor(1-\varepsilon) \lambda_{1} F_{1}+\cdots+(1-\varepsilon) \lambda_{r} F_{r}-K_{\pi}\right\rfloor .
$$


Using the same arguments that we used in the proof of Theorem 3.4 we may provide the following formula for the multiplicity of a jumping point in terms of the minimal jumping divisor.

Proposition 4.4. Let $\mathfrak{a}=\left(\mathfrak{a}_{1}, \ldots, \mathfrak{a}_{r}\right) \subseteq\left(\mathcal{O}_{X, O}\right)^{r}$ be a tuple of $\mathfrak{m}$-primary ideals and $G_{\boldsymbol{\lambda}}$ the maximal jumping divisor associated to some jumping point $\boldsymbol{\lambda} \in \mathbb{R}_{>0}^{r}$. Then,

$$
m(\boldsymbol{\lambda})=\left(\left\lceil K_{\pi}-\lambda_{1} F_{1}-\cdots-\lambda_{r} F_{r}\right\rceil+G_{\boldsymbol{\lambda}}\right) \cdot G_{\boldsymbol{\lambda}}+\#\left\{\text { connected components of } G_{\boldsymbol{\lambda}}\right\}
$$

It was proved in [4, Lemma 4.6] that two interior points of a $\mathcal{C}$-facet have the same minimal jumping divisor, which we refer to as the minimal jumping divisor associated to the $\mathcal{C}$-facet. Therefore by applying 4.4, the multiplicity is constant along the interior points of a $\mathcal{C}$-facet and thus proving Proposition 4.2. We point out that two interior points of a $\mathcal{C}$-facet may have different maximal jumping divisor

This constancy property for the multiplicities is no longer true when considering jumping points at the intersection of $\mathcal{C}$-facets. However we can control the multiplicity depending on the number of $\mathcal{C}$-facets that contain this jumping point.

Theorem 4.5. Let $\mathfrak{a}=\left(\mathfrak{a}_{1}, \ldots, \mathfrak{a}_{r}\right) \subseteq\left(\mathcal{O}_{X, O}\right)^{r}$ be a tuple of $\mathfrak{m}$-primary ideals and let $L: \boldsymbol{c}_{0}+\mu \mathbf{u}$ and $L^{\prime}: \boldsymbol{c}_{0}^{\prime}+\mu \mathbf{u}$ be two parallel rays that are close enough. Let $\boldsymbol{\lambda} \in L$ be a jumping point and $B_{\varepsilon}(\boldsymbol{\lambda})$ be a ball centered at $\boldsymbol{\lambda}$ of a sufficiently small radius $\varepsilon>0$ such that $L \cap \mathbf{J W}_{\mathfrak{a}} \cap B_{\varepsilon}(\boldsymbol{\lambda})=\{\boldsymbol{\lambda}\}$. If $L^{\prime} \cap \mathbf{J W}_{\mathfrak{a}} \cap B_{\varepsilon}(\boldsymbol{\lambda})=\left\{\boldsymbol{\lambda}_{1}, \ldots, \boldsymbol{\lambda}_{n}\right\}$ then

$$
m(\boldsymbol{\lambda})=m\left(\boldsymbol{\lambda}_{1}\right)+m\left(\boldsymbol{\lambda}_{2}\right)+\cdots+m\left(\boldsymbol{\lambda}_{n}\right) \text {. }
$$

Proof. Let $V_{1}, \ldots, V_{k}$ be all the hyperplanes associated to exceptional divisors that contain the jumping point $\boldsymbol{\lambda}$. For each hyperplane $V_{i}$ we consider the divisor $H_{i}=\sum_{j} E_{j}$ where the sum is taken over the exceptional divisors that support the hyperplane $V_{i}$, i.e. for all $E_{j} \leq H_{i}$ there exists some $\ell_{j} \in \mathbb{Z}_{>0}$ such that the hyperplane $V_{i}$ is of the form $e_{1, j} z_{1}+\cdots+e_{r, j} z_{r}=\ell_{j}+k_{j}$. Notice that, even though it is possible that not all of these hyperplanes support a jumping wall, we have a decomposition of the maximal jumping divisor as $H_{\boldsymbol{\lambda}}=H_{1}+\cdots+H_{k}$. Let $\left\{\mathbf{c}_{1}, \ldots, \mathbf{c}_{k}\right\}$ be the ordered ${ }^{2}$ set of points resulting from the intersection of the ray $L^{\prime}$ with the hyperplanes $V_{1}, \ldots, V_{k}$. Notice that we have $L^{\prime} \cap \mathbf{J W}_{\mathfrak{a}} \cap B_{\varepsilon}(\boldsymbol{\lambda})=\left\{\boldsymbol{\lambda}_{1}, \ldots, \boldsymbol{\lambda}_{n}\right\} \subseteq\left\{\mathbf{c}_{1}, \ldots, \mathbf{c}_{k}\right\}$.

For each $\mathbf{c}_{i}=\left(c_{i, 1}, \ldots, c_{i, r}\right)$ we may find a point $\left(1-\varepsilon^{\prime}\right) \mathbf{c}_{i}:=\left(\left(1-\varepsilon_{1}^{\prime}\right) c_{i, 1}, \ldots,\left(1-\varepsilon_{r}^{\prime}\right) c_{i, r}\right)$ over the ray $L^{\prime}$ that is close enough but smaller than $\mathbf{c}_{1}$ and a point over the ray $L$ and smaller than $\boldsymbol{\lambda}$ that we will denote as $(1-\varepsilon) \boldsymbol{\lambda}:=\left(\left(1-\varepsilon_{1}\right) \lambda_{1}, \ldots,\left(1-\varepsilon_{r}\right) \lambda_{r}\right)$ satisfying

$$
\left\lceil K_{\pi}-\left(1-\varepsilon_{1}\right) \lambda_{1} F_{1}-\cdots-\left(1-\varepsilon_{r}\right) \lambda_{r} F_{r}\right\rceil=\left\lceil K_{\pi}-\left(1-\varepsilon_{1}^{\prime}\right) c_{i, 1} F_{1}-\cdots-\left(1-\varepsilon_{r}^{\prime}\right) c_{i, r} F_{r}\right\rceil .
$$

From the construction of the hyperplanes $V_{i}$ we have:

$$
\begin{aligned}
& \cdot\left\lceil K_{\pi}-\lambda_{1} F_{1}-\cdots-\lambda_{r} F_{r}\right\rceil=\left\lceil K_{\pi}-\left(1-\varepsilon_{1}\right) \lambda_{1} F_{1}-\cdots-\left(1-\varepsilon_{r}\right) \lambda_{r} F_{r}\right\rceil+H_{\lambda} . \\
& \cdot\left\lceil K_{\pi}-c_{i, 1} F_{1}-\cdots-c_{i, r} F_{r}\right\rceil=\left\lceil K_{\pi}-\left(1-\varepsilon_{1}^{\prime}\right) c_{i, 1} F_{1}-\cdots-\left(1-\varepsilon_{r}^{\prime}\right) c_{i, r} F_{r}\right\rceil+H_{1}+\cdots+H_{i} .
\end{aligned}
$$

${ }^{2}$ The order on the set of points $\left\{\mathbf{c}_{1}, \ldots, \mathbf{c}_{k}\right\}$ is given by their distance to the origin. We order the hyperplanes $V_{1}, \ldots, V_{k}$ accordingly. 
Therefore

$$
\left\lceil K_{\pi}-\lambda_{1} F_{1}-\cdots-\lambda_{r} F_{r}\right\rceil-\left\lceil K_{\pi}-c_{i, 1} F_{1}-\cdots-c_{i, r} F_{r}\right\rceil=H_{i+1}+\cdots H_{k} .
$$

By Theorem 3.4, one has

$$
m(\boldsymbol{\lambda})=\left(\left\lceil K_{\pi}-\lambda_{1} F_{1}-\cdots-\lambda_{r} F_{r}\right\rceil+H_{\boldsymbol{\lambda}}\right) \cdot H_{\boldsymbol{\lambda}}+\#\left\{\text { connected components of } H_{\boldsymbol{\lambda}}\right\} .
$$

Thus, we can rewrite this formula as

$$
\begin{aligned}
m(\boldsymbol{\lambda})= & \sum_{i=1}^{k}\left(\left\lceil K_{\pi}-\lambda_{1} F_{1}-\cdots-\lambda_{r} F_{r}\right\rceil+H_{i}\right) \cdot H_{i} \\
& +\sum_{i=1}^{k} \sum_{\substack{j=1 \\
j \neq i}}^{k} H_{i} H_{j}+\#\left\{\text { connected components of } H_{\boldsymbol{\lambda}}\right\} \\
= & \sum_{i=1}^{k}\left(\left\lceil K_{\pi}-c_{i, 1} F_{1}-\cdots-c_{i, r} F_{r}\right\rceil+H_{i}\right) \cdot H_{i} \\
& +\sum_{i=1}^{k} \sum_{j>i}^{k} H_{i} H_{j}+\#\left\{\text { connected components of } H_{\boldsymbol{\lambda}}\right\}
\end{aligned}
$$

where the last equality follows from Equation 4.1. Now, recall that for any divisor $D$ with exceptional support $\#\{$ connected components of $D\}=v_{D}-a_{D}$, where $v_{D}$ and $a_{D}$ denote the number of vertices and edges of $D$ in the dual graph. Since $v_{H_{\boldsymbol{\lambda}}}=v_{H_{1}}+\cdots+v_{H_{k}}$ and $a_{H_{\boldsymbol{\lambda}}}=a_{H_{1}}+\cdots+a_{H_{k}}+\sum_{i=1}^{k} \sum_{j>i}^{k} H_{i} H_{j}$ we deduce

$$
\#\left\{\text { connected components of } H_{\boldsymbol{\lambda}}\right\}=\sum_{i=1}^{k} \#\left\{\text { connected components of } H_{i}\right\}-\sum_{i=1}^{k} \sum_{j>i}^{k} H_{i} H_{j} \text {. }
$$

Therefore

$$
\begin{aligned}
m(\boldsymbol{\lambda}) & =\sum_{i=1}^{k}\left[\left(\left\lceil K_{\pi}-c_{i, 1} F_{1}-\cdots-c_{i, r} F_{r}\right\rceil+H_{i}\right) \cdot H_{i}+\#\left\{\text { connected components of } H_{i}\right\}\right] \\
& =m\left(\mathbf{c}_{1}\right)+\cdots+m\left(\mathbf{c}_{k}\right) .
\end{aligned}
$$

The only points with non zero multiplicity are those over a jumping wall, namely the jumping points $\left\{\boldsymbol{\lambda}_{1}, \ldots, \boldsymbol{\lambda}_{n}\right\}$ and thus we get the desired result.

\section{Contribution to the log-CANonical Wall}

Let $X$ be a smooth complex surface and $\mathfrak{a} \subseteq \mathcal{O}_{X, O}$ an ideal. A common theme in the study of multiplier ideals is to check which exceptional divisors contribute to the jumping 
numbers of $\mathfrak{a}$. In the case of the log-canonical threshold we know that it is described by the formula

$$
\operatorname{lct}(\mathfrak{a})=\min _{i}\left\{\frac{k_{i}+1}{e_{i}}\right\} .
$$

In the case that $\mathfrak{a}$ is $\mathfrak{m}$-primary and simple, this minimum is achieved at the first rupture or dicritical exceptional component, starting from the origin, in the dual graph of the log-resolution of $\mathfrak{a}$ (see [15], [23]). For non simple ideals we may find some analogous statements in [16], [13], [6], [5].

For the case of mixed multiplier ideals, Cassou-Noguès and Libgober [11, Theorem 4.22 ] studied the contribution of exceptional divisors to the log-canonical wall for the case where the tuple of ideals corresponds to the branches of a plane curve. In this section we will give a generalization of their result that works for general tuples of $\mathfrak{m}$-primary ideals $\mathfrak{a}=\left(\mathfrak{a}_{1}, \ldots, \mathfrak{a}_{r}\right) \subseteq\left(\mathcal{O}_{X, O}\right)^{r}$, where $X$ is a complex surface with a rational singularity at $O$ and the points in the log-canonical wall have multiplicity one.

Their result is described in terms of the so-called Newton nest introduced in [11, Definition 4.19]. In order to give a generalization to our setup of the Newton nest we will need to fix some notation. When $X$ has a rational singularity we may have an strict inclusion $\mathcal{O}_{X, O} \supsetneq \mathcal{J}\left(\mathfrak{a}^{\mathbf{0}}\right)$ where $\mathbf{0}=(0, \ldots, 0)$ is the origin of the positive orthant $\mathbb{R}_{\geqslant 0}^{r}$. Indeed, the mixed multiplier ideal $\mathcal{J}\left(\mathbf{a}^{\mathbf{0}}\right)$ is described by a divisor $D_{\mathbf{0}}=\sum e_{j}^{\mathbf{0}} E_{j}$ which is the antinef closure of $\left\lfloor-K_{\pi}\right\rfloor$ that can be computed using the unloading procedure described in [2]. Therefore, the log-canonical wall is supported on hyperplanes of the form

$$
e_{1, j} z_{1}+\cdots+e_{r, j} z_{r}=k_{j}+1+e_{j}^{\mathbf{0}}, \quad j=1, \ldots, s .
$$

For each point $z_{i}=\left(0, \ldots, 0, \operatorname{lct}\left(\mathfrak{a}_{i}\right), 0, \ldots, 0\right)$ in the $i$-th coordinate axis corresponding to the $\log$-canonical threshold of the ideal $\mathfrak{a}_{i}, i=1, \ldots, r$, we consider the reduced divisor $G_{\boldsymbol{z}_{i}}^{\prime}=\sum E_{j}$, where the sum is taken over those exceptional divisors associated to the supporting hyperplanes of the log-canonical wall which contain the point $z_{i}$. Notice that this divisor is contained in the minimal jumping divisor of $z_{i}$, that is $G_{z_{i}}^{\prime} \leqslant G_{z_{i}}$.

Definition 5.1. Consider the minimal connected subgraph $\Gamma_{\mathfrak{a}}^{\prime}$ of the dual graph $\Gamma_{\mathfrak{a}}$ containing the divisors $G_{\boldsymbol{z}_{i}}^{\prime}$, for $i=1 \ldots, r$. The Newton nest of $\Gamma_{\mathfrak{a}}$ is the set of rupture or dicritical divisors belonging to $\Gamma_{\mathfrak{a}}^{\prime}$.

Remark 5.2. In the case that $X$ is smooth and the ideals $\mathfrak{a}_{i}$ are simple, this definition coincides with the one given by Cassou-Noguès and Libgober in [11, Definition 4.19] since in this case we have that $G_{\boldsymbol{z}_{i}}^{\prime}=G_{\boldsymbol{z}_{i}}=E_{j_{i}}$, where $E_{j_{i}}$ is the rupture divisor in the dual graph $\Gamma_{\mathfrak{a}_{i}}$ which is closest to its root.

Cassou-Noguès and Libgober [11, Theorem 4.22] established a one-to-one correspondence between the divisors of the Newton nest and the $\mathcal{C}$-facets of the log-canonical wall in the case where $X$ is smooth and the tuple of ideals correspond to the branches of a plane curve. The only restriction that we are going to impose in our generalization is that the multiplicity of all the points in the log-canonical wall have multiplicity one. This 
condition is achieved, for example, in the case that $X$ has a log-terminal singularity at $O \in X$.

Lemma 5.3. Let $\mathfrak{a}=\left(\mathfrak{a}_{1}, \ldots, \mathfrak{a}_{r}\right) \subseteq\left(\mathcal{O}_{X, O}\right)^{r}$ be a tuple of simple $\mathfrak{m}$-primary ideals and $X$ is a complex surface with a log-terminal singularity. Then, all the points in the logcanonical wall have multiplicity one.

Proof. From the definition of log-terminal singularity, it follows that the antinef closure of $\left\lfloor-K_{\pi}\right\rfloor$ is 0 because all the coefficients of $\left\lfloor-K_{\pi}\right\rfloor$ are strictly smaller than one. Therefore the ideal associated to the point $\mathbf{0}$ is the whole ring.

Let $\boldsymbol{\lambda}$ be a jumping point in the log-canonical wall. All the coefficients of the divisor $\left\lfloor\lambda F-K_{\pi}\right\rfloor$ must be smaller or equal to one so we have $\left\lfloor\lambda F-K_{\pi}\right\rfloor \leqslant Z$ where $Z$ is the fundamental cycle. Therefore we have $\mathfrak{m}=\pi_{*} \mathcal{O}_{X^{\prime}}(-Z) \subseteq \mathcal{J}\left(\mathfrak{a}^{\boldsymbol{\lambda}}\right) \subsetneq \mathcal{O}_{X, O}$. So $\mathcal{J}\left(\mathfrak{a}^{\boldsymbol{\lambda}}\right)=\mathfrak{m}$, and consequently $m(\boldsymbol{\lambda})=1$ for all points in the log-canonical wall.

Before stating the main result of this section we will present some properties concerning jumping points of multiplicity one. This is a very restrictive condition on the corresponding minimal jumping divisors. To such purpose we have to introduce some technical notation. Given any exceptional component $E_{i}$ and a reduced divisor $D \leqslant E=\operatorname{Exc}(\pi)$, we define the set of components adjacent to $E_{i}$ inside $D$ and its number as:

$$
\operatorname{Adj}_{D}\left(E_{i}\right)=\left\{E_{j} \leqslant D \mid E_{i} \cdot E_{j}=1\right\} \quad \text { and } a_{D}\left(E_{i}\right)=\# \operatorname{Adj}_{D}\left(E_{i}\right)
$$

Lemma 5.4. Let $\mathfrak{a}=\left(\mathfrak{a}_{1}, \ldots, \mathfrak{a}_{r}\right) \subseteq\left(\mathcal{O}_{X, O}\right)^{r}$ be a tuple of $\mathfrak{m}$-primary ideals and $\boldsymbol{\lambda}$ a jumping point such that $m(\boldsymbol{\lambda})=1$. Then, the minimal jumping divisor $G_{\boldsymbol{\lambda}}$ has only one connected component and no rupture or dicritical divisor $E_{i}$ such that $a_{G_{\lambda}}\left(E_{i}\right)>1$.

Proof. Using Proposition 4.4 we have the following formula.

$$
m(\boldsymbol{\lambda})=\left(\left\lceil K_{\pi}-\lambda_{1} F_{1}-\cdots-\lambda_{r} F_{r}\right\rceil+G_{\boldsymbol{\lambda}}\right) \cdot G_{\boldsymbol{\lambda}}+\#\left\{\text { connected components of } G_{\boldsymbol{\lambda}}\right\} .
$$

In the case that $m(\boldsymbol{\lambda})=1$ we can deduce that $\#$ connected components of $\left.G_{\boldsymbol{\lambda}}\right\}=1$ and

$$
\left(\left\lceil K_{\pi}-\lambda_{1} F_{1}-\cdots-\lambda_{r} F_{r}\right\rceil+G_{\boldsymbol{\lambda}}\right) \cdot G_{\boldsymbol{\lambda}}=\sum_{E_{i} \leqslant G_{\boldsymbol{\lambda}}}\left(\left\lceil K_{\pi}-\lambda_{1} F_{1}-\cdots-\lambda_{r} F_{r}\right\rceil+G_{\boldsymbol{\lambda}}\right) \cdot E_{i}=0
$$

since we already had $\left(\left\lceil K_{\pi}-\lambda_{1} F_{1}-\cdots-\lambda_{r} F_{r}\right\rceil+G_{\boldsymbol{\lambda}}\right) \cdot G_{\boldsymbol{\lambda}} \geqslant 0$ by [4, Proposition 4.13]. Indeed, using again this result we have

$$
\left(\left\lceil K_{\pi}-\lambda_{1} F_{1}-\cdots-\lambda_{r} F_{r}\right\rceil+G_{\boldsymbol{\lambda}}\right) \cdot E_{i}=0
$$

for all $E_{i} \leqslant G_{\boldsymbol{\lambda}}$. We may provide a more explicit description of this equation using [4, Lemma 4.11]. Namely we have

$$
\begin{aligned}
& \left(\left\lceil K_{\pi}-\lambda_{1} F_{1}-\cdots-\lambda_{r} F_{r}\right\rceil+G_{\boldsymbol{\lambda}}\right) \cdot E_{i}= \\
& \quad=-2+\lambda_{1} \rho_{1, i}+\cdots+\lambda_{r} \rho_{r, i}+a_{G_{\boldsymbol{\lambda}}}\left(E_{i}\right)+\sum_{E_{j} \in \operatorname{Adj}_{E}\left(E_{i}\right)}\left\{\lambda_{1} e_{1, j}+\cdots+\lambda_{r} e_{r, j}-k_{j}\right\} .
\end{aligned}
$$


Thus, if $E_{i}$ is a rupture or dicritical component with $a_{G_{\boldsymbol{\lambda}}}\left(E_{i}\right)>1$, then we have

$$
\left(\left\lceil K_{\pi}-\lambda_{1} F_{1}-\cdots-\lambda_{r} F_{r}\right\rceil+G_{\boldsymbol{\lambda}}\right) \cdot E_{i}>0
$$

so we get a contradiction and the result follows.

Corollary 5.5. Let $\boldsymbol{\lambda} \in \mathbb{R}_{\geqslant 0}^{r}$ be a jumping point not contained in any coordinate hyperplane such that $m(\boldsymbol{\lambda})=1$. Then:

i) If $\boldsymbol{\lambda}$ is an interior point of a $\mathcal{C}$-facet which does not intersect any other $\mathcal{C}$-facet, the minimal jumping divisor $G_{\boldsymbol{\lambda}}$ contains at most two dicritical or rupture divisors.

ii) If $\boldsymbol{\lambda}$ is an interior point of a $\mathcal{C}$-facet which intersects, at least, another $\mathcal{C}$-facet, the minimal jumping divisor $G_{\boldsymbol{\lambda}}$ is a dicritical or rupture divisor.

iii) If $\boldsymbol{\lambda}$ is at the intersection of two $\mathcal{C}$-facets, the minimal jumping divisor $G_{\boldsymbol{\lambda}}$ is connected and contains exactly two dicritical or rupture divisors, which are its two ends.

Proof. Let $\lambda \in \mathbb{R}_{\geqslant 0}^{r}$ be a jumping point. By [4, Theorem 4.14], the ends of the connected components of the minimal jumping divisor $G_{\boldsymbol{\lambda}}$ over the dual graph are either rupture or dicritical divisors. If we assume $m(\boldsymbol{\lambda})=1$, then, using Lemma 5.4, we have that $a_{G_{\boldsymbol{\lambda}}}\left(E_{j}\right) \leqslant 1$ for any rupture or dicritical divisor $E_{j}$. Therefore either $G_{\boldsymbol{\lambda}}$ is just one exceptional component or it is connected with just two ends which are rupture or dicritical divisors in the dual graph. In particular, i) follows.

Now assume that $\boldsymbol{\lambda}$ is at the intersection of two $\mathcal{C}$-facets $\mathcal{C}_{1}$ and $\mathcal{C}_{2}$ with associated minimal jumping divisors $G_{1}$ and $G_{2}$ respectively. We have $G_{\boldsymbol{\lambda}}=G_{1}+G_{2}+G^{\prime}$ for some divisor $G^{\prime}$ with exceptional support. Moreover, the jumping points in the interior of $\mathcal{C}_{1}$ and $\mathcal{C}_{2}$ have multiplicity 1 so the same properties considered above also apply for $G_{1}$ and $G_{2}$. The two $\mathcal{C}$-facets are supported on different hyperplanes with different slope, so $G_{1}$ and $G_{2}$ do not share any exceptional divisor. By Lemma 5.4, this forces $G_{1}$ and $G_{2}$ to be just one exceptional component being a rupture or dicritical divisor and the minimal jumping divisor $G_{\boldsymbol{\lambda}}$ contains exactly two dicritical or rupture divisors. Thus, ii) and iii) follow.

Remark 5.6. Given a tuple of $\mathfrak{m}$-primary ideals $\mathfrak{a}=\left(\mathfrak{a}_{1}, \ldots, \mathfrak{a}_{r}\right) \subseteq\left(\mathcal{O}_{X, O}\right)^{r}$ we may pick a subfamily $\mathfrak{a}^{\prime}=\left\{\mathfrak{a}_{i_{1}}, \ldots, \mathfrak{a}_{i_{k}} \mid 1 \leq i_{1}<\cdots<i_{k} \leq r\right\}$ and, if no confusion arise, we may view it either as a tuple $\left(\mathcal{O}_{X, O}\right)^{s}$ or a subtuple of $\mathfrak{a}$ in $\left(\mathcal{O}_{X, O}\right)^{r}$ in the obvious way. Notice for example that the Newton nest of $\mathfrak{a}^{\prime}$ is a subset of the Newton nest of $\mathfrak{a}$. In the case that $\boldsymbol{\lambda}=\left(\lambda_{1}, \ldots, \lambda_{r}\right) \in \mathbb{R}_{\geqslant 0}^{r}$ is a jumping point contained in a coordinate hyperplane, we may consider the tuple $\mathfrak{a}^{\prime}=\left(\mathfrak{a}_{i} \mid \lambda_{i} \neq 0\right)$. Corollary 5.5 holds whenever we consider $\boldsymbol{\lambda}$ as a jumping point for $\mathfrak{a}^{\prime}$ and thus, a point not in the coordinate hyperplanes of the lower dimensional positive orthant.

Notice that Corollary 5.5 already singles out a very particular case where we may not have our desired one-to-one correspondence. Namely, assume that the log-canonical wall has a unique $\mathcal{C}$-facet with points of multiplicity one. Part i) of Corollary 5.5 says 
that the Newton nest contains either one or two divisors. Therefore the desired one-toone correspondence fails when we have exactly two divisors and this case can indeed be achieved. Recall that the effective divisors $F_{i}$ such that $\mathfrak{a}_{i} \cdot \mathcal{O}_{X^{\prime}}=\mathcal{O}_{X^{\prime}}\left(-F_{i}\right)$ are of the form $F_{i}=\sum_{j=1}^{s} e_{i, j} E_{j}$ for $i=1, \ldots, r$ and the relative canonical divisor is $K_{\pi}=\sum_{i=1}^{s} k_{j} E_{j}$. Let $E_{j}$ and $E_{\ell}$ be the divisors in the Newton nest and $V_{j, 1}: e_{1, j} z_{1}+\cdots+e_{r, j} z_{r}=k_{j}+1$ and $V_{\ell, 1}: e_{1, \ell} z_{1}+\cdots+e_{r, \ell} z_{r}=k_{\ell}+1$ be their associated hyperplanes. The numerical conditions for which these hyperplanes support the unique $\mathcal{C}$-facet of the log-canonical wall are

$$
\frac{e_{1, \ell}}{e_{1, j}}=\cdots=\frac{e_{r, \ell}}{e_{r, j}}=\frac{k_{\ell}+1}{k_{j}+1} .
$$

This result can be reformulated in the following

Lemma 5.7. Let $\mathfrak{a}=\left(\mathfrak{a}_{1}, \ldots, \mathfrak{a}_{r}\right) \subseteq\left(\mathcal{O}_{X, O}\right)^{r}$ be a tuple of $\mathfrak{m}$-primary ideals where $X$ is a complex surface with a rational singularity at $O \in X$. Assume that all the points in the log-canonical wall have multiplicity one and the Newton nest contains two divisors $E_{j}$ and $E_{\ell}$. Then, the log-canonical wall has a unique $\mathcal{C}$-facet if and only if

$$
\frac{e_{1, \ell}}{e_{1, j}}=\cdots=\frac{e_{r, \ell}}{e_{r, j}}=\frac{k_{\ell}+1}{k_{j}+1} .
$$

We illustrate this case with the following

Example 5.8. Consider a smooth surface $X$ and a tuple of ideals $\mathfrak{a}=\left\{\mathfrak{a}_{1}, \mathfrak{a}_{2}\right\}$ such that they have a minimal log-resolution with the following vertex ordering

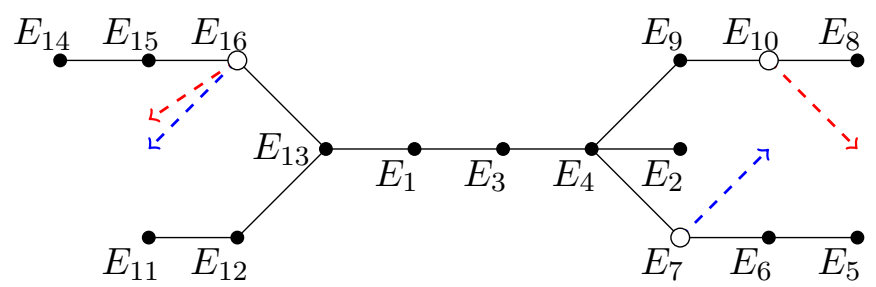

and the divisors given by the ideals are:

$\cdot F_{1}=(18,24,45,72,73,146,218,72,144,216,21,42,63,64,128,194)$, and

- $F_{2}=(18,24,45,72,72,144,216,74,147,222,21,42,63,64,128,194)$.

with $K_{\pi}=(1,2,4,7,8,16,24,8,16,25,2,4,6,7,14,21)$.

The divisors in the Newton nest are $E_{4}$ and $E_{13}$ and the log-canonical wall only has a unique $\mathcal{C}$-facet whose supporting hyperplane has the following equation:

$$
V_{4,1}: 72 x_{1}+72 x_{2}=8 \text {, or equivalently, } V_{13,1}: 63 x_{1}+63 x_{2}=7 \text {. }
$$

The main result of this section is that the one-to-one correspondence established by Cassou-Noguès and Libgober in [11, Theorem 4.22] still holds in our setup except for this very particular case where we may have two divisors in the Newton nest and just a unique $\mathcal{C}$-facet. 
Theorem 5.9. Let $\mathfrak{a}=\left(\mathfrak{a}_{1}, \ldots, \mathfrak{a}_{r}\right) \subseteq\left(\mathcal{O}_{X, O}\right)^{r}$ be a tuple of $\mathfrak{m}$-primary ideals where $X$ is a complex surface with a rational singularity at $O \in X$. Assume that the log-canonical wall has at least two $\mathcal{C}$-facets and all its points have multiplicity one. Then, there is a one-to-one correspondence between the exceptional divisors in the Newton nest of $\mathfrak{a}$ and the $\mathcal{C}$-facets of the log-canonical wall.

Proof. We start with the case $r=2$, that is $\mathfrak{a}=\left(\mathfrak{a}_{1}, \mathfrak{a}_{2}\right)$. In order to construct the Newton nest of $\mathfrak{a}$ we start considering two points $z_{1}$ and $z_{2}$ in the coordinate axes corresponding to the log-canonical thresholds of the ideals $\mathfrak{a}_{1}$ and $\mathfrak{a}_{2}$ respectively. Then we order the $\mathcal{C}$-facets of the log-canonical wall $\mathcal{C}_{1}, \mathcal{C}_{2}, \ldots, \mathcal{C}_{l}$ in such a way that $\boldsymbol{z}_{1} \in \mathcal{C}_{1}, \boldsymbol{z}_{2} \in \mathcal{C}_{l}$ and each $\mathcal{C}$-facet $\mathcal{C}_{i}$ intersects $\mathcal{C}_{i-1}$ and $\mathcal{C}_{i+1}$. Roughly speaking, we are considering a path from $z_{1}$ to $z_{2}$ over the log-canonical wall. By Corollary 5.5, the exceptional divisors associated to the supporting hyperplanes of $\mathcal{C}_{1}, \mathcal{C}_{2}, \ldots, \mathcal{C}_{l}$ are unique, but they are also different because the hyperplanes have different slopes. Therefore, we can order these dicritical or rupture divisors $E_{j_{1}}, E_{j_{2}}, \ldots, E_{j_{l}}$ accordingly to their corresponding $\mathcal{C}$-facets. Moreover, Corollary 5.5 implies that they form a path in the dual graph $\Gamma_{\mathfrak{a}}$ of the log-resolution of $\mathfrak{a}$ with no dicritical or rupture divisors in between two consecutive $E_{j_{i}}$ 's.

Now, let $\Gamma_{\mathfrak{a}}^{\prime} \subset \Gamma_{\mathfrak{a}}$ be the minimal connected subgraph containing $E_{j_{1}}$ and $E_{j_{s}}$. Then we have that $E_{j_{2}}, \ldots, E_{j_{s-1}}$ belong to $\Gamma_{\mathfrak{a}}^{\prime}$ and the result follows because the Newton nest is the set $\left\{E_{j_{1}}, E_{j_{2}}, \ldots, E_{j_{s}}\right\}$ by construction.

For the case $r>2$, that is $\mathfrak{a}=\left(\mathfrak{a}_{1}, \ldots, \mathfrak{a}_{r}\right)$, we have to consider points $\boldsymbol{z}_{1}, \ldots, \boldsymbol{z}_{r}$ in the coordinate axes corresponding to the log-canonical thresholds. We are going to pick one of them, say $\boldsymbol{z}_{1}$, and a $\mathcal{C}$-facet $\mathcal{C}_{1}$ containing this point $\boldsymbol{z}_{1}$. Let $\mathbf{c} \in \mathcal{C}_{1}$ be an interior point with rational coordinates and $\mathbf{q}:=\left(0, q_{2}, \ldots, q_{r}\right)$ a point in the coordinate hyperplane $\left\{x_{1}=0\right\}$ with rational coordinates. Notice that $\mathbf{q}$ corresponds to the mixed multiplier ideal $\mathcal{J}\left(\mathfrak{a}_{2}^{q_{2}} \cdots \mathfrak{a}_{r}^{q_{r}}\right)=\mathcal{J}\left(\left(\mathfrak{a}_{2}^{d q_{i}} \cdots \mathfrak{a}_{r}^{d q_{r}}\right)^{\frac{1}{d}}\right)$ for some $d \in \mathbb{Z}$ such that $d q_{i} \in \mathbb{Z}$ for $i=2, \ldots, r$.

The mixed multiplier ideals appearing in the restriction of the positive orthant $\mathbb{R}_{\geqslant 0}^{r}$ to the plane containing the points $\mathbf{c}, \mathbf{q}$ and the origin $\mathbf{0}$ are the mixed multiplier ideals of the duple $\mathfrak{a}^{\prime}=\left(\mathfrak{a}_{1}, \mathfrak{a}_{2}^{d q_{2}} \cdots \mathfrak{a}_{r}^{d q_{r}}\right)$ so the facets of the corresponding log-canonical wall are in one-to-one correspondence with the exceptional divisors in the Newton nest of $\mathfrak{a}^{\prime}$ which is contained in the Newton nest of $\mathfrak{a}$. Just moving the points $\mathbf{c}$ and $\mathbf{q}$ conveniently allows us to cover the whole log-canonical wall of $\mathfrak{a}$ and the result follows. We point out that a $\log$-resolution of $\mathfrak{a}$ is also a log-resolution of $\mathfrak{a}^{\prime}$.

In the following example we show that with our definition of the Newton nest we may also consider the case of non-simple ideals in a smooth surface which was not considered in [11].

Example 5.10. Consider the tuple of ideals $\mathfrak{a}=\left(\mathfrak{a}_{1}, \mathfrak{a}_{2}, \mathfrak{a}_{3}\right)$ on a smooth surface $X$ :

$$
\begin{aligned}
\cdot \mathfrak{a}_{1}= & \left(y^{3}, x^{6} y, x^{8}, x^{3} y^{2}\right), \\
\cdot \mathfrak{a}_{2}= & \left(x\left(x^{2}+x+y\right)^{4},\left(x^{2}+x+y\right)^{2}\left(x^{2}-x-y\right)^{2}, x^{5}\left(x^{2}-x-y\right)^{2}, x^{5}\left(x^{2}+x+y\right)^{2},\right. \\
& \left.x^{3}\left(x^{2}+x+y\right)\left(x^{2}-x-y\right)^{2}, x^{3}\left(x^{2}+x+y\right)^{3}\right),
\end{aligned}
$$


$\cdot \mathfrak{a}_{3}=\left(-x^{6}(x-y), x^{7}, x^{5}(x-y)^{2},-x^{3}(x-y)^{3}, x^{5}(x-y)^{2},-(x-y)^{5}, x^{2}(x-y)^{4}\right)$.

The dual graph of the log-resolution of $\mathfrak{a}$ is:

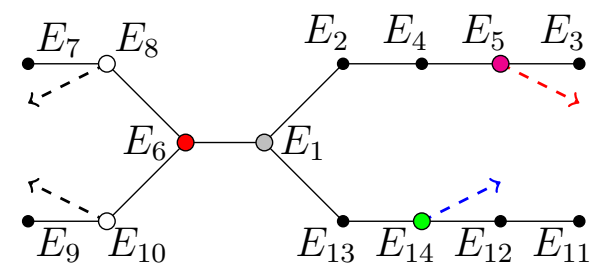

For simplicity we denote the divisors associated with this resolution as

- $F_{1}=(3,6,8,15,24,3,3,6,3,6,3,6,9,15)$,

- $F_{2}=(4,4,4,8,12,8,9,18,9,18,4,8,12,20)$,

$\cdot F_{3}=(5,5,5,10,15,5,5,10,5,10,7,14,20,35)$.

In the same manner, the relative canonical divisor is $K_{\pi}=(1,2,3,6,10,2,3,6,3,6,2,4,6,10)$. The Newton nest of $\mathfrak{a}$ consists of the exceptional divisors $E_{1}, E_{5}, E_{6}$ and $E_{14}$ and they correspond, matching the colors, to the $\mathcal{C}$-facets of the log-canonical wall which is:

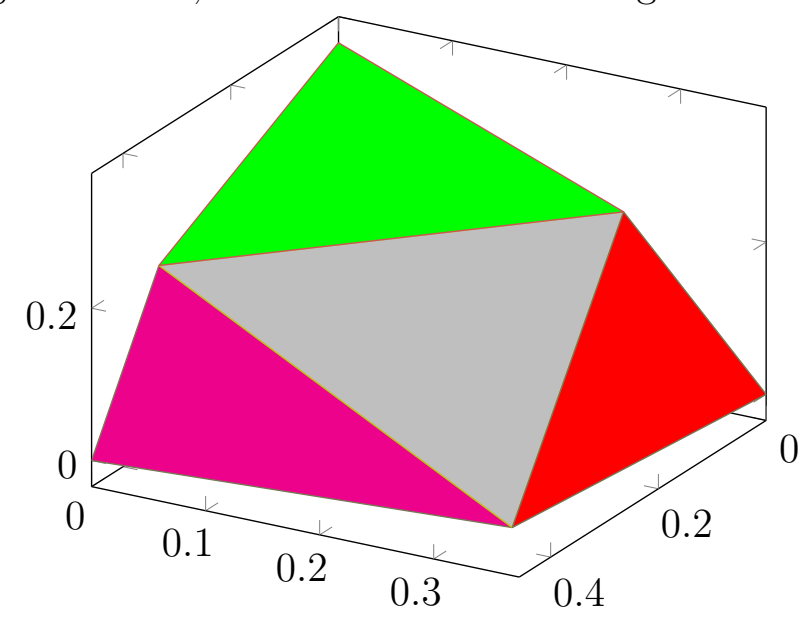

In the following example we present a case where $X$ has a log-canonical singularity and Theorem 5.9 does not hold. This shows how sharp is the condition of having points in the log-canonical wall with multiplicity one.

Example 5.11. Consider a surface $X$ with a rational singularity at $O$ whose minimal resolution $\pi: X^{\prime} \longrightarrow X$ has six exceptional components $E_{1}, \ldots, E_{6}$ with the following dual graph and intersection matrix:

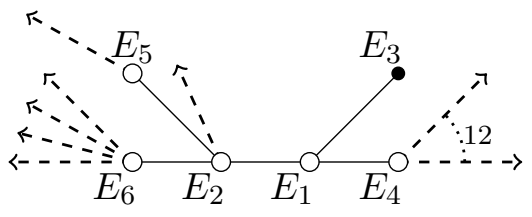

Vertex ordering 


$$
M=\left(E_{i} \cdot E_{j}\right)_{1 \leqslant i, j \leqslant 6}=\left(\begin{array}{cccccc}
-2 & 1 & 1 & 1 & 0 & 0 \\
1 & -3 & 0 & 0 & 1 & 1 \\
1 & 0 & -1 & 0 & 0 & 0 \\
1 & 0 & 0 & -3 & 0 & 0 \\
0 & 1 & 0 & 0 & -3 & 0 \\
0 & 1 & 0 & 0 & 0 & -6
\end{array}\right) .
$$

The fundamental cycle is the divisor $Z=(3,2,3,1,1,1)$ and the relative canonical divisor is $K_{\pi}=\left(-\frac{1}{2},-1, \frac{1}{2},-\frac{1}{2},-\frac{2}{3},-\frac{5}{6}\right)$ so the singularity is log-canonical. Then we consider a duple of ideals $\mathfrak{a}=\left(\mathfrak{a}_{1}, \mathfrak{a}_{2}\right)$, with $\mathfrak{a}_{1}$ non singular and $\mathfrak{a}_{2}=\mathfrak{m}$ given by the divisors $F_{1}=(15,6,15,9,2,1)$ and $F_{2}=Z=(3,2,3,1,1,1)$. The log-canonical wall has two $\mathcal{C}$-facets and the corresponding mixed multiplier ideals are different (see Figure 2). In particular we have jumping points on the log-canonical wall with multiplicity bigger that 1 . In this case the Newton nest consists of the exceptional divisors $E_{1}, E_{2}$ and $E_{4}$ so we no longer have the bijection given in Theorem 5.9.

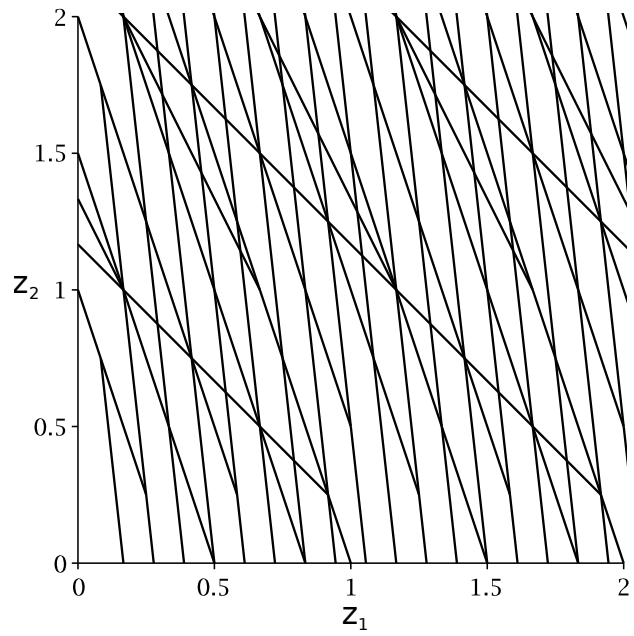

Figure 2. Constancy regions of the the mixed multiplier ideals of $\mathfrak{a}$.

\section{REFERENCES}

[1] M. Alberich-Carramiñana, J.Àlvarez Montaner and G. Blanco, Monomial generators of complete planar ideals, preprint available at arXiv:1701.03503. 9

[2] M. Alberich-Carramiñana, J. Àlvarez Montaner and F. Dachs-Cadefau, Multiplier ideals in twodimensional local rings with rational singularities, Mich. Math. J. 65 (2016), 287-320. 13

[3] M. Alberich-Carramiñana, J. Àlvarez Montaner, F. Dachs-Cadefau and V. González-Alonso, Poincaré series of multiplier ideals in two-dimensional local rings with rational singularities, Adv. Math. 304 (2017), 769-792. 2, 4, 5, 7 
[4] M. Alberich-Carramiñana, J. Àlvarez Montaner and F. Dachs-Cadefau, Constancy regions of mixed multiplier ideals in two-dimensional local rings with rational singularities, Math. Nachr. 291 (2018), 245-263. 3, 4, 5, 9, 10, 11, 14, 15

[5] M. Aprodu and D. Naie, Enriques diagrams and log-canonical thresholds of curves on smooth surfaces, Geom. Dedicata 146 (2010), 43-66. 13

[6] E. Artal Bartolo, Pi Cassou-Noguès, I. Luengo and A. Melle-Hernández, On the log-canonical threshold for germs of plane curves, Singularities I, 1-14, Contemp. Math., 474, Amer. Math. Soc., 2008. 13

[7] M. Artin, Some numerical criteria for contractability of curves on algebraic surfaces, Amer. J. Math. 84 (1962), 485-496.

[8] M. Artin, On isolated rational singularities of surfaces, Amer. J. Math. 68 (1966), 129-136. 3

[9] G. Blanco and F. Dachs-Cadefau Computing multiplier ideals in smooth surfaces. In: Research Perspectives. Positivity and Valuations. CRM. Barcelona. In press. 9

[10] Pi. Cassou-Noguès and A. Libgober, Multivariable Hodge theoretical invariants of germs of plane curves, J. Knot Theory Ramifications 20 (2011), 787-805. 2

[11] Pi. Cassou-Noguès and A. Libgober, Multivariable Hodge theoretical invariants of germs of plane curves II, in Valuation Theory in Interaction. Eds. A Campillo, F.-V. Kuhlmann and B. Teissier. EMS Series of Congress Reports 10 (2014), 82-135. 2, 3, 13, 16, 17

[12] L. Ein, R. Lazarsfeld, K. Smith and D. Varolin, Jumping coefficients of multiplier ideals, Duke Math. J. 123 (2004), 469-506. 1

[13] C. Galindo, F. Hernando and F. Monserrat, The log-canonical threshold of a plane curve, Math. Proc. Cambridge Math. Soc. 160 (2016), 513-535. 13

[14] C. Galindo and F. Monserrat, The Poincaré series of multiplier ideals of a simple complete ideal in a local ring of a smooth surface, Adv. Math. 225 (2010), 1046-1068. 2, 7

[15] T. Järviletho, Jumping numbers of a simple complete ideal in a two-dimensional regular local ring, Mem. Amer. Math. Soc. 214 (2011), no. 1009, viii+78 pp. 13

[16] T. Kuwata, On log canonical thresholds of reducible plane curves, Amer. J. Math. 121 (1999), 701-721. 13

[17] R. Lazarsfeld, Positivity in algebraic geometry. II, volume 49, (2004), Springer-Verlag, xviii+385. 5

[18] A. Libgober, Hodge decomposition of Alexander invariants, Manuscripta Math. 107 (2002) 251-269. 2

[19] A. Libgober and M. Mustaţă, Sequences of LCT-polytopes, Math. Res. Lett. 18 (2011) 733-746. 2

[20] J. Lipman, Rational singularities, with applications to algebraic surfaces and unique factorization, Inst. Hautes tudes Sci. Publ. Math. 36 (1969) 195-279. 3

[21] D. Naie, Mixed multiplier ideals and the irregularity of abelian coverings of smooth projective surfaces, Expo. Math. 31 (2013), 40-72. 2

[22] A. J. Reguera, Curves and proximity on rational surface singularities, J. Pure Appl. Algebra 122 (1997) 107-126. 3

[23] K. Tucker, Jumping numbers on algebraic surfaces with rational singularities, Trans. Amer. Math. Soc. 362 (2010), 3223-3241. 13 
Deptartament de Matemàtiques, Universitat Politècnica de Catalunya, Av. Diagonal 647, Barcelona 08028, Spain

E-mail address: Maria.Alberich@upc.edu, Josep.Alvarez@upc.edu

Institut für Mathematik, Martin-Luther-Universität Halle-WittenberG 06099 Halle (SaAle), Germany

E-mail address: Ferran.Dachs-Cadefau@mathematik.uni-halle.de

Institut für Algebraische Geometrie, Leibniz Universität Hannover, Welfengarten 1, 30167 Hannover, Germany

E-mail address: gonzalez@math.uni-hannover.de 